\title{
FOURIER TRANSFORMS OF CERTAIN CLASSES OF INTEGRABLE FUNCTIONS(1)
}

\author{
BY \\ ROBERT RYAN
}

1. Introduction. Let $G$ be an arbitrary locally compact abelian group with character group $\hat{G}$. This paper is devoted to characterizing those functions $\phi \in L_{\infty}(\hat{G})$ which are equal almost everywhere (a.e.) to the Fourier tranform of some function $f \in L_{1}(G) \cap L_{p}(G)$ where $1 \leqq p \leqq \infty$. The characterizations presented originate from the following theorem by I. J. Schoenberg [12]:

THEOREM 1. Let $V(-\infty, \infty)$ denote the set of all functions of bounded variation on the real line, and for $\mu \in V(-\infty, \infty)$ define $\hat{\mu}$ by $\hat{\mu}(x)$ $=\int_{-\infty}^{\infty} e^{-i x y} d \mu(y)$. If $\phi \in L_{\infty}(-\infty, \infty)$ then $\phi(x)=\hat{\mu}(x)$ a.e. for some $\mu \in V(-\infty, \infty)$ if and only if there exists a constant $K>0$ such that

$$
\left|\int_{-\infty}^{\infty} f(x) \phi(x) d x\right| \leqq K \sup _{-\infty<x<\infty}\left|\int_{-\infty}^{\infty} e^{-i x y} f(y) d y\right|
$$

for all $f \in L_{1}(-\infty, \infty)$.

This theorem characterizes those functions $\phi \in L_{\infty}(-\infty, \infty)$ of the form $\phi(x)=\hat{\mu}(x)$ a.e. in terms of a continuity condition on the linear functional defined by $F(f)=\int_{-\infty}^{\infty} f(x) \phi(x) d x$ for $f \in L_{1}(-\infty, \infty)$. Given a subset $N \subset$ $V(-\infty, \infty)$ it is possible to ask whether additional continuity conditions on $F$ can be found which, combined with the above condition, are necessary and sufficient in order that $\phi(x)=\hat{\mu}(x)$ a.e. for some $\mu \in N$. The following theorem by A. C. Berry [1] illustrates such a condition when $N$ is the class absolutely continuous functions of bounded variation. Here we define

$$
\hat{f}(x)=\int_{-\infty}^{\infty} e^{-i x y} f(y) d y \text { for } f \in L_{1}(-\infty, \infty)
$$

Presented to the Society, November 19, 1960; received by the editors October 11, 1960, and, in revised form, October 6, 1961.

(1) This paper is taken from my doctoral dissertation written under the direction of Professor W.A.J. Luxemburg at the Claifornia Institute of Technology. The original work was done while I was a National Science Foundation Cooperative Graduate Fellow. The preparation for publication and the revision was partially supported by N. S. F. Grant No. G-14002 and by the U. S. Army's Mathematical Research Center, University of Wisconsin under contract DA-11-022-ORD-2059. 
THEOREM 2. Suppose that $\phi \in L_{\infty}(-\infty, \infty)$. The following conditions are necessary and sufficient in order that $\phi(x)=\hat{g}(x)$ a.e. for some $g \in L_{1}(-\infty, \infty)$ :

(1) There exists a constant $K>0$ such that $|F(f)| \leqq K\|\hat{f}\|_{\infty}$ for all $f \in L_{1}(-\infty, \infty)$.

(2) For every $\varepsilon>0$ there exists a $\delta=\delta(\varepsilon)>0$ such that $|F(f)| \leqq \varepsilon\|\hat{f}\|_{\infty}$ whenever $f \in L_{1}(-\infty, \infty), \hat{f} \in L_{1}(-\infty, \infty)$ and $\|\hat{f}\|_{1} \leqq \delta\|\hat{f}\|_{\infty}$.

Condition (1) is just a restatement of Theorem 1; it is this part of the hypothesis which insures that $\phi$ be equal a.e. to the Fourier transform of some $\mu \in V(-\infty, \infty)$. Condition (2) implies that $\mu$ is absolutely continuous.

A theorem similar to Berry's theorem was previously proved for the circle group by R. Salem $[10 ; 11]$.

THEOREM 3. Let $(Z)$ be the class of functions $\omega(x)=\sum_{n=1}^{\infty}\left(\alpha \cos n x+\beta_{n} \sin n x\right)$ which are continuous and differentiable with $|\omega(x)|<1$ and such that the Fourier series of $\omega^{\prime}$ is absolutely convergent. The following conditions are necessary and sufficient in order that $\left(a_{n}, b_{n}\right)$ be the Fourier coefficients of an integrable function:

(A) The formally integrated series $\sum_{n=1}^{\infty}\left(a_{n} n^{-1} \sin n x-b_{n} n^{-1} \cos n x\right)$ converges to a continuous function.

(B) The expression $\sum_{n=1}^{\infty}\left(a_{n} \alpha_{n}+b_{n} \beta_{n}\right)$ tends to zero when $\omega$ varies in $(Z)$ in such a way that $\sum_{n=1}^{\infty}\left(\alpha_{n}^{2}+\beta_{n}^{2}\right)$ tends to zero.

Here condition (A) implies that $\left(-b_{n} n^{-1}, a_{n} n^{-1}\right)$ is the set of Fourier coefficients of some continuous function. Condition (B) implies that this function is absolutely continuous and hence an integral of some function $f \in L_{1}(0,2 \pi)$. It then follows that $\left(a_{n}, b_{n}\right)$ is the set of Fourier coefficients for $f$.

In this paper we prove for an arbitrary locally compact abelian group a theorem which gives as a special case the second part of Berry's theorem. This, combined with the generalization of Schoenberg's theorem, constitutes a characterization of the Fourier transforms of $L_{1}(G)$. We also include a slightly different statement and proof of Salem's theorem. Using the same ideas we characterize the Fourier transforms of $L_{1}(G) \cap L_{p}(G)$ for $1<p \leqq \infty$. The statement and proof of Theorem 1 generalize directly for a locally compact abelian group (see Eberlein [3]). Thus, in the statements of our theorems we will generally assume that $\phi$, the function under investigation, is equal almost everywhere to the Fourier transform of some bounded Radon measure $\mu$. Here we are concerned with the additional conditions which $\phi$ must satisfy in order that $d \mu(x)=f(x) d x$ for some $f \in L_{1}(G) \cap L_{p}(G)$.

$\S 2$ contains notation and definitions; $\S 3$ treats $L_{1}(G) ; \S 4$ is devoted to $L_{1}(G) \cap L_{p}(G)$ for $1<p \leqq \infty$.

The author wishes to thank the referee for the very simple proof of Lemma 1 and the useful comments on the proof of Theorem 5. 
2. Preliminaries. Throughout this paper $G$ denotes an arbitrary locally compact abelian group, and the group operation is denoted by + . The character group of $G$ is denoted by $\hat{G}$. The complex number $(x, \hat{x})$ is the value of the character $\hat{x} \in \hat{G}$ at the point $x \in G$.

The space of all bounded, continuous, complex valued functions defined on $G$ is denoted by $C(G)$. We give $C(G)$ the usual norm:

$$
\|f\|_{\infty}=\sup _{x \in G}|f(x)|
$$

$f \in C(G)$.

$C_{\infty}(G)$ denotes the subspace of functions $f \in C(G)$ which vanish at infinity, i.e, for each $f \in C_{\infty}(G)$ and $\varepsilon>0$ there exists a compact set $A \subset G$ such that $|f(x)|<\varepsilon$ for $x \in A^{\prime}$ (= the complement of $A$ ). $C_{\infty \infty}(G)$ denotes the set of functions in $C_{\infty}(G)$ which have compact support.

Let $\mathscr{B}$ denote the smallest $\sigma$-algebra of subsets of $G$ containing the compact subsets(2), and let $M(G)$ denote the space of all complex valued, bounded, regular and countably additive set functions defined on $\mathscr{B} . M(G)$ is identified with the space of all bounded linear functionals on $C_{\infty}(G)$, and an element $\mu \in M(G)$ is called a bounded Radon measure. For detailed discussions of $M(G)$ and references we refer the reader to the survey articles by Hewitt [7] and Rudin [9].

Let $m$ be a nontrivial Haar measure defined on $\mathscr{B}$. $L_{p}(G)$ for $1 \leqq p<\infty$ denotes the space of all $\mathscr{B}$-measurable, complex valued functions defined on $G$ for which

$$
\|f\|_{p}=\left(\int_{G}|f(x)|^{p} d m(x)\right)^{1 / p}<+\infty .
$$

$L_{\infty}(G)$ denotes the space of $\mathscr{B}$-measurable functions such that

$$
\|f\|_{\infty}=\inf \{\alpha \mid m\{x|x \in G,| f(x) \mid>\alpha\}=0\}<+\infty .
$$

(If $f \in C(G)$ then the two definitions of $\|f\|_{\infty}$ agree.)

There corresponds to each $f \in L_{1}(G)$ a unique measure $\mu_{f} \in M(G)$ defined by $\mu_{f}(E)=\int_{E} f(x) d x$ for all $E \in \mathscr{B}\left({ }^{3}\right)$. By the Radon-Nikodym theorem $\mu=\mu_{f}$ for some $f \in L_{1}(G)$ if and only if $\mu$ is absolutely continuous with respect to $m$.

The convolution of two functions $f \in L_{1}(G)$ and $g \in L_{p}(G)$ with $1 \leqq p \leqq \infty$ is defined in the usual way:

$$
f * g(x)=\int_{G} f(x-y) g(y) d y .
$$

This integral exists for almost every $x \in G$ and defines a function $f * g \in L_{p}(G)$ with $\|f * g\|_{p} \leqq\|f\|_{1}\|g\|_{p}$. If $\left\{u_{\alpha} \mid \alpha \in \mathscr{A}\right\}$ is an approximate identity for the

(2) See Halmos [5] for measure theoretic terminology not explained here.

(3) The differential of Haar measure will be written $d x, d y$ etc. 
algebra $L_{1}(G)$ then it is well known that $\lim _{\alpha}\left\|u_{\alpha} * f-f\right\|_{p}=0$ for all $f \in L_{p}(G)$ with $1 \leqq p<\infty$. (See Loomis [8].) Since $G$ is a locally compact Hausdorff space we may assume that $u_{\alpha} \in C_{\infty \infty}(G)$ for all $\alpha \in \mathscr{A}$. If $f \in C(G)$ is uniformly continuous it is easy to show that $\lim _{\alpha} u_{\alpha} * f(x)=f(x)$ uniformly for $x \in G$.

The following notation will be used for the various Fourier transforms:

$$
\begin{aligned}
& \hat{\mu}(\hat{x})=\int_{G}(-x, \hat{x}) d \mu(x), \quad \mu \in M(G) . \\
& \hat{f}(\hat{x})=\int_{G}(-x, \hat{x}) f(x) d x, \quad f \in L_{1}(G) .
\end{aligned}
$$

If $g \in L_{1}(\hat{G})$ we will write

$$
\hat{g}(x)=\int_{\hat{G}}(x, \hat{x}) g(\hat{x}) d \hat{x} .
$$

The Haar measure on $G$ is normalized so that $\int_{G}|f(x)|^{2} d x=\int_{\hat{G}}|\hat{f}(\hat{x})|^{2} d \hat{x}$ for $f \in L_{1}(G) \cap L_{2}(G)$. If $N \subset M(G)$ then $N^{\wedge}$ denotes the set of functions $\hat{\mu}$ where $\mu \in N$.

$P(G)$ denotes the set of continuous, positive definite functions defined on $G$, and $\left[L_{1}(G) \cap P(G)\right]$ denotes the linear space spanned by $L_{1}(G) \cap P(G)$. If $f \in\left[L_{1}(G) \cap P(G)\right]$ then $\hat{f}=g \in L_{1}(\hat{G})$ and $f(x)=\hat{g}(x)$ for all $x \in G$ [8]. From this it is seen that $\left[L_{1}(G) \cap P(G)\right]^{\wedge}=\left[L_{1}(\hat{G}) \cap P(\hat{G})\right]$. A simple argument using an approximate identity shows that $\left[L_{1}(G) \cap P(G)\right]$ is dense in $L_{p}(G)$ for $1 \leqq p<\infty$. Since $\left(L_{1}(\hat{G})\right)^{\wedge}$ is dense in $C_{\infty}(G)$ it follows that $\left[L_{1}(G) \cap P(G)\right]=\left[L_{1}(\hat{G}) \cap P(\hat{G})\right]^{\wedge}$ is dense in $C_{\infty}(G)$.

3. $L_{1}(G)$. Throughout this section we assume that $\phi \in L_{\infty}(\hat{G})$ is of the form $\phi(\hat{x})=\hat{\mu}(\hat{x})$ a.e. for some $\mu \in M(G)$. Theorems 4 and 5 present respectively necessary and sufficient conditions on $\phi$ in order that $\phi(\hat{x})=\hat{f}(\hat{x})$ a.e. for some $f \in L_{1}(G)$. A special case of these theorems, combined with the generalization of Theorem 1, gives Berry's theorem.

LEMMA 1. If $f \in L_{1}(G)$ then the linear functional defined by

$$
F(g)=\int_{G} g(x) f(x) d x
$$

for $g \in L_{\infty}(G)$ satisfies the following condition;

For every $p$ with $1 \leqq p<\infty$ and every $\varepsilon>0$ there exists a $\delta>0$ depending only upon $p, \varepsilon$ and $f$ and such that

$$
|F(g)| \leqq \varepsilon\|g\|_{\infty}
$$

whenever $g \in L_{p}(G) \cap L_{\infty}(G)$ and

$$
\|g\|_{p} \leqq \delta\|g\|_{\infty} .
$$

Proof. Let $p$ and $\varepsilon$ be given and fixed. Assume that $\|g\|_{\infty}=1$. Then we must find a $\delta>0$ such that $\|g\|_{p} \leqq \delta$ implies $|F(g)| \leqq \varepsilon$. If this were not possible 
there would exist a sequence of functions $\left\{g_{n}\right\}$ with $\left\|g_{n}\right\|_{\infty}=1,\left\|g_{n}\right\|_{p}<1 / n$ and $\left|F\left(g_{n}\right)\right|>\varepsilon>0$. Then there exists a subsequence $\left\{g_{n_{k}}\right\}$ such that $g_{n_{k}} \rightarrow 0$ a.e. on the set where $f(x) \neq 0$. Hence by the theorem on dominated convergence $F\left(g_{n_{k}}\right)=\int_{G} g_{n_{k}}(x) f(x) d x$ tends to zero. This contradiction proves the lemma.

THEOREM 4. If $\phi=\hat{f}$ a.e. for some $f \in L_{1}(G)$ then the linear functional defined for $g \in L_{1}(\hat{G})$ by

$$
F(g)=\int_{\widehat{G}} g(\hat{x}) \phi(\hat{x}) d \hat{x}
$$

satisfies the following condition:

For every $p$ with $1 \leqq p<\infty$ and every $\varepsilon>0$ there exists a $\delta>0$ depending only upon $\varepsilon, p$ and $\phi$ and such that

$$
|F(g)| \leqq \varepsilon\|\hat{g}\|_{\infty}
$$

whenever $g \in\left[L_{1}(\hat{G}) \cap P(\hat{G})\right]$ and

$$
\|\hat{g}\|_{p} \leqq \delta\|\hat{g}\|_{\infty} .
$$

Proof. If $g \in\left[L_{1}(\hat{G}) \cap P(\hat{G})\right]$ then $\hat{g} \in\left[L_{1}(G) \cap P(G)\right]$ and hence $g \in L_{p}(G) \cap$ $L_{\infty}(G)$. By Fubini's theorem

$$
F(g)=\int_{\hat{G}} g(\hat{x}) \phi(\hat{x}) d \hat{x}=\int_{G} \hat{g}(x) f(-x) d x .
$$

The result now follows from the lemma.

THEOREM 5. Suppose $\phi=\hat{\mu}$ a.e. for some $\mu \in M(G)$. Then $\phi=\hat{f}$ a.e. for some $f \in L_{1}(G)$ if the functional defined by

$$
F(g)=\int_{\hat{G}} g(\hat{x}) \phi(\hat{x}) d \hat{x}
$$

for $g \in L_{1}(\hat{G})$ satifies the following condition:

There exists a $p$ with $1 \leqq p<\infty$ such that for every $\varepsilon>0$ there is a $\delta>0$ depending only upon $\varepsilon, p$ and $\phi$ and such that

$$
|F(g)| \leqq \varepsilon\|\hat{g}\|_{\infty}
$$

whenever $g \in\left[L_{1}(\hat{G}) \cap P(\hat{G})\right]$ and

$$
\|\hat{g}\|_{p} \leqq \delta\|\hat{g}\|_{\infty} .
$$

Proof. If $g \in\left[L_{1}(\hat{G}) \cap P(\hat{G})\right]$ then by Fubini's theorem

$$
F(g)=\int_{\hat{G}}(\hat{x}) \phi(\hat{x}) d \hat{x}=\int_{G} \hat{g}(-x) d \mu(x) .
$$

Hence it is sufficient to show that if $\mu$ is not absolutely continuous then there exists a sequence of functions $\left\{g_{n}\right\}$ with $g_{n} \in\left[L_{1}(G) \cap P(G)\right]=\left[L_{1}(\hat{G}) \cap P(\hat{G})\right]^{\wedge}$ 
and such that $\left\|g_{n}\right\|_{\infty} \rightarrow 1,\left\|g_{n}\right\|_{p} \rightarrow 0$ but such that $\int_{G} g_{n}(x) d \mu(x)$ does not tend to zero as $n \rightarrow \infty$. Here $p$ is an arbitrary number with $1 \leqq p<\infty$. It is also sufficient to assume that $\mu$ is a real valued measure since we will make the functions $g_{n}$ real valued.

Now assume that $\mu$ has a nontrivial singular part $\lambda$. In general $\|\lambda\|$ $=\sup \left\{\left|\int_{G} h(x) d \lambda(x)\right| \mid h \in C_{\infty \infty}(G), h\right.$ is a real valued and $\left.\|h\|_{\infty}=1\right\}$. However since $\lambda$ is singular with respect to the Haar measure $m$ the supremum may be taken over just those functions for which $m$ (support of $h$ ) $\leqq \eta$ where $\eta$ is an arbitrary positive number. In order to see this assume that $\lambda$ is positive. The general case involves considering the positive and negative variations of $\lambda$. Then since $\lambda$ is regular $\|\lambda\|=\sup \{\lambda(K) \mid K \subset G$ is compact, $m(K)=0\}$ where $\lambda(K)=\inf \left\{\int_{G} g(x) d \lambda(x) \mid g \in C_{\infty \infty}(G), g(x)=1\right.$ for $x \in K$ and $\left.0 \leqq g(x) \leqq 1\right\}$. Since $m(K)=0$ it is clear that the functions in this last expression may be taken with $m$ (support of $g$ ) $\leqq, \eta>0$ and $\eta$ independent of $K$.

Thus for every $n=1,2,3, \ldots$ there exists a real valued function $h_{n} \in C_{\infty \infty \infty}(G)$ such that $\left\|h_{n}\right\|_{\infty}=1,\|\lambda\|-\left|\int_{G} h_{n}(x) d \lambda(x)\right| \leqq n^{-1}$ and $m$ (support of $h_{n}$ ) $n^{-1}$. For each $h_{n}$ we can choose a real valued function $u_{n} \in C_{\infty \infty}(G)$ from an approxmate identity such that

$$
\left|h_{n}(x)-h_{n} * u_{n}(x)\right| \leqq n^{-1} .
$$

The functions $g_{n}=h_{n} * u_{n}$ then have the required properties. In particular being a convolution of functions in $C_{\infty \infty}(G), g_{n} \in\left[L_{1}(G) \cap P(G)\right]$ and

$$
\left.\left\|g_{n}\right\|_{p} \leqq\left\|h_{n}\right\|_{p}\left\|u_{n}\right\|_{1}=\left\|h_{n}\right\|_{p} \leqq\left[m \text { (support of } h_{n}\right)\right]^{1 / p} \leqq n^{-1 / p} \text {. }
$$

From $\left|h_{n}(x)-g_{n}(x)\right| \leqq n^{-1}$ it follows that

Writing

$$
\left\|g_{n}\right\|_{\infty} \rightarrow 1 \text { and } \int_{G} g_{n}(x) d \lambda(x) \rightarrow\|\lambda\| \text { as } n \rightarrow \infty \text {. }
$$

$$
\int_{G} g_{n}(x) d \mu(x)=\int_{G} g_{n}(x) d \lambda(x)+\int_{G} g_{n}(x) d(\mu-\lambda)(x)
$$

we see that $\int_{G} g_{n}(x) d \mu(x) \rightarrow\|\lambda\| \neq 0$ since by Theorem 4 the second integral tends to zero. This proves the theorem.

Theorems 4 and 5 combined with Theorem 1 reduce to Berry's theorem (Theorem 2) by letting $G$ be the real line and $p=1$.

We now apply Theorem 5 to prove Salem's theorem with slightly weaker hypothesis. The notation is the same as in the statement of Theorem 3.

THEOREM 6. Suppose that $-b_{n} n^{-1}=\frac{1}{2 \pi} \int_{0}^{2 \pi} f(x) \cos n x d x, \quad a_{n} n^{-1}=\frac{1}{2 \pi} \int_{0}^{2 \pi} f(x) \sin n x d x, \quad n=1,2, \ldots$, for some continuous function $f$ defined on the unit circle. Then $f$ is an absolutely continuous function of bounded variation and 


$$
a_{n}=\frac{1}{2 \pi} \int_{0}^{2 \pi} f^{\prime}(x) \cos n x d x, \quad b_{n}=\frac{1}{2 \pi} \int_{0}^{2 \pi} f^{\prime}(x) \sin n x d x
$$

if and only if condition (B) of Theorem 3 holds.

Proof. We will prove only the sufficiency of the condition.

Let $Z$ be the class of all functions $g(x)=\sum_{n=1}^{\infty}\left(\alpha_{n} \cos n x+\beta_{n} \sin n x\right)$ for which $g^{\prime}$ exists and has an absolutely convergent Fourier series. Then $g \in(Z)$ if and only if $g \in Z$ and $\|g\|_{\infty} \leqq 1$.

For $g \in Z$ define the linear functional $F$ by

$$
F(g)=-\frac{1}{2 \pi} \int_{0}^{2 \pi} f(x) g^{\prime}(x) d x=\sum_{n=1}^{\infty}\left(a_{n} \alpha_{n}+b_{n} \beta_{n}\right) .
$$

The continuity condition (B) and the fact that

$$
\sum_{n=1}^{\infty}\left(\alpha_{n}^{2}+\beta_{n}^{2}\right)=\frac{1}{2 \pi} \int_{0}^{2 \pi}|g(x)|^{2} d x \leqq\|g\|_{\infty}^{2}
$$

imply immediately that $F$ is a bounded linear functional on $Z$. Since $Z$ is dense in $C(0,2 \pi) F$ can be uniquely extended to a bounded linear functional $F$ defined on $C(0,2 \pi)$. By the Riesz representation theorem for linear functional of $C(0,2 \pi)$ there exists a function of bounded variation $\alpha$ such that

$$
F(g)=\frac{1}{2 \pi} \int_{0}^{2 \pi} g(x) d \alpha(x), \quad g \in C(0,2 \pi) .
$$

Letting $g(x)$ be $\cos n x$ and $\sin n x$ gives

$$
a_{n}=\frac{1}{2 \pi} \int_{0}^{2 \pi} \cos n x d \alpha(x) \text { and } b_{n}=\int_{0}^{2 \pi} \sin n x d \alpha(x) .
$$

This proves that $\left(a_{n}, b_{n}\right)$ is the set of Fourier coefficients of a function of bounded variation. The theorem now follows from Theorem 5 by taking $G$ to be the circle group and $p=2$ and observing that if condition (B) holds for $g \in Z$ then it must also hold for $g \in\left[L_{1}(0,2 \pi) \cap P(0,2 \pi)\right]$.

4. $L_{1}(G) \cap L_{p}(G)$ for $1<p \leqq \infty$. Presented in this section are two necessary and sufficient conditions on $\phi \in L_{\infty}(\hat{G})$ in order that $\phi=\hat{f}$ a.e. for some $f \in$ $L_{1}(G) \cap L_{p}(G)$ : Theorem 7 gives a continuity condition on the functional $F(g)$ $=\int_{\hat{G}} g(\hat{x}) \phi(\hat{x}) d \hat{x}$ for $g \in L_{1}(\hat{G})$; Theorem 9 presents a multiplier condition on $\phi$.

THEOREM 7. Suppose that $\phi=\hat{\mu}$ a.e. for some $\mu \in M(G)$ and define

$$
F(g)=\int_{\hat{G}} g(\hat{x}) \phi(\hat{x}) d \hat{x}
$$

for $g \in L_{1}(\hat{G})$. In order that $\phi=\hat{f}$ a.e. for some $f \in L_{1}(G) \cap L_{p}(G)$ with $1<p \leqq \infty$ it is necessary and sufficient that there exist a constant $K>0$ such that 


$$
|F(g)| \leqq K\|\hat{g}\|_{q}
$$

for all $g \in\left[L_{1}(\hat{G}) \cap P(\hat{G})\right]$ where $1 / p+1 / q=1$.

Proof. A proof of the necessity of the condition is readily constructed by using the techniques of the sufficiency proof in reverse. We therefore proceed directly to a proof of the sufficiency.

Assume that there exists a $K>0$ such that

$$
|F(g)| \leqq K\|\hat{g}\|_{q} \text { for all } g \in\left[L_{1}(\hat{G}) \cap P(\hat{G})\right] .
$$

Define the linear functional $H$ on $\left[L_{1}(\hat{G}) \cap P(\hat{G})\right]^{\wedge}=\left[L_{1}(G) \cap P(G)\right]$ by

$$
H(\hat{g})=F(g) \text {. }
$$

Since Fourier transforms are unique $H$ is well defined. Furthermore

$$
|H(g)| \leqq K\|g\|_{q}
$$

for all $g \in\left[L_{1}(G) \cap P(G)\right]$. This shows that $H$ is a bounded linear functional defined on a dense subset of $L_{q}(G)$, and hence $H$ can be extended uniquely to all of $L_{q}(G)$ without changing its norm. Let $\bar{H}$ be the extension of $H$. Then there exists a unique function $f \in L_{p}(G)$ such that

$$
\bar{H}(g)=\int_{G} g(x) f(-x) d x
$$

for all $g \in L_{q}(G)$. If $g \in\left[L_{1}(\hat{G}) \cap P(\hat{G})\right]$

$$
F(g)=\int_{\widehat{G}} g(\hat{x}) \phi(\hat{x}) d \hat{x}=\int_{\widehat{G}} g(\hat{x})\left[\int_{G}(-x, \hat{x}) d \mu(x)\right] d \hat{x}=\int_{G} \hat{g}(-x) d \mu(x) .
$$

Since $F(g)=\bar{H}(\hat{g})$ we have

$$
\int_{G} \hat{g}(-x) d \mu(x)=\int_{G} \hat{g}(x) f(-x) d x=\int_{G} \hat{g}(-x) f(x) d x
$$

for all $\hat{g} \in\left[L_{1}(\hat{G}) \cap P(\hat{G})\right]^{\wedge}=\left[L_{1}(G) \cap P(G)\right]$. The fact that $\left[L_{1}(G) \cap P(G)\right]$ is dense in $L_{q}(G)$ and $C_{\infty}(G)$ implies that

$$
\int_{G} g(x) d \mu(x)=\int_{G} g(x) f(x) d x
$$

for all $g \in C_{\infty \infty \infty}(G)$. From this it follows that

$$
\|f\|_{1}=\sup \left\{\left|\int_{G} g(x) f(x) d x\right| \mid g \in C_{\infty \infty}(G),\|g\|_{\infty} \leqq 1\right\}<+\infty .
$$

Thus $f \in L_{1}(G)$ as well as $L_{p}(G)$, and $\hat{\mu}(\hat{x})=\hat{f}(\hat{x})$ for all $\hat{x} \in \hat{G}$. This proves the theorem.

If $G$ is compact it is not necessary to assume that $\phi=\hat{\mu}$ a.e. for some $\mu \in M(G)$. In this case $\|\hat{g}\|_{q} \leqq\|\hat{g}\|_{\infty}$ for $g \in L_{1}(\hat{G})$, and the condition $|F(g)| \leqq K\|\hat{g}\|_{q}$ 
implies that $|F(g)| \leqq K\|\hat{g}\|_{\infty}$. Since for $G$ compact $L_{1}(\hat{G})=\left[L_{1}(\hat{G}) \cap P(\hat{G})\right]$ this holds for all $g \in L_{1}(\hat{G})$. The generalization of Theorem 1 then insures tha $\phi=\hat{\mu}$ for some $\mu \in M(G)$.

In the case that $G$ is compact Theorem 7 implies the Riesz-Fisher theorem. For let $\phi$ be an element of $L_{2}(\hat{G})$. Then if $g \in\left[L_{1}(\hat{G}) \cap P(\hat{G})\right]$ we have $|F(g)|$ $\leqq\|\phi\|_{2}\|g\|_{2}=\|\phi\|_{2}\|\hat{g}\|_{2}$, and from Theorem $7, \phi=\hat{f}$ for some $f \in L_{1}(G) \cap$ $L_{2}(G)=L_{2}(G)$.

The next theorem is related to certain older results by $\mathrm{H}$. Cramér [2]. We omit the proof since it is almost a direct consequence of Theorem 7 .

THEOREM 8. Let $\left\{\hat{f}_{\alpha}\right\}$ be a net of Fourier transforms with $f_{\alpha} \in L_{1}(G) \cap L_{p}(G)$ where $1<p \leqq \infty$, and such that $\left\|f_{\alpha}\right\|_{p} \leqq K<+\infty$. If $\phi=\hat{\mu}$ a.e. for some $\mu \in M(G)$ and if

$$
\lim _{\alpha} \int_{\hat{G}} g(\hat{x}) f_{\alpha}(\hat{x}) d \hat{x}=\int_{\hat{G}} g(\hat{x}) \phi(\hat{x}) d \hat{x}
$$

for all $g \in\left[L_{1}(\hat{G}) \cap P(\hat{G})\right]$ then $\phi=\hat{f}$ a.e. for some $f \in L_{1}(G) \cap L_{p}(G)$.

The final theorem is an addition to the extensive literature on multipliers or factor functions. The proof is modeled after the proof of a similar theorem by Helson [6].

THEOREM 9. Suppose that $\phi \in L_{\infty}(\hat{G})$. Then $\phi=\hat{f}$ a.e. for some $f \in L_{1}(G) \cap$ $L_{p}(G)$ with $1<p \leqq \infty$ if and only if $\phi \cdot \hat{g} \in\left(L_{1}(G) \cap L_{p}(G)\right)^{\wedge}$ for all $g \in L_{1}(G)$.

Proof. The necessity of the condition is just the fact that $f * g \in L_{1}(G) \cap L_{p}(G)$ and $(f * g)^{\wedge}=\hat{f} \cdot \hat{g}$. To show the sufficiency we first observe that the condition implies that $\phi \cdot \hat{g} \in\left(L_{1}(G)\right)^{\wedge}$ for all $g \in L_{1}(G)$. Thus by Helson's theorem [6] $\phi=\hat{\mu}$ a.e. for some $\mu \in M(G)$. This $\mu$ defines a bounded linear transformation $g \rightarrow \mu * g$ of $L_{1}(G)$ into $L_{1}(G)$ with

$$
\|\mu * g\|_{1} \leqq\|\mu\|\|g\|_{1}, \quad g \in L_{1}(G) .
$$

The condition of the theorem implies by means of the closed graph theorem that this transformation is also bounded from $L_{1}(G)$ into $L_{p}(G)$. Thus there exists a constant $K>0$ such that

$$
\left\|\mu^{*} g\right\|_{p} \leqq K\|g\|_{1}, g \in L_{1}(G) .
$$

Ler $\left\{u_{\alpha}\right\}$ be an approximate identity for $L_{1}(G)$. Then for $g \in\left[L_{1}(\hat{G}) \cap P(\hat{G})\right]$

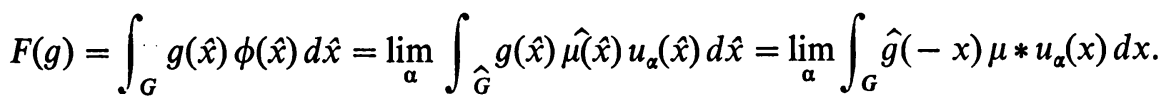

Combining the above inequalities give

$$
|F(g)| \leqq \lim _{\alpha}\left\|\mu * u_{\alpha}\right\|_{p}\|\hat{g}\|_{q} \leqq \lim _{\alpha} K\left\|u_{\alpha}\right\|_{1}\|\hat{g}\|_{q}, \text { and since }\left\|u_{\alpha}\right\|_{1}=1
$$


we get

$$
|F(g)| \leqq K\|\hat{g}\|_{q}
$$

for all $g \in\left[L_{1}(\hat{G}) \cap P(\hat{G})\right]$. The result now follows directly from Theorem 7 .

Theorem 9 can also be proved by using a result of Edwards [4] concerning the form of bounded linear transformations from $L_{1}(G)$ into $L_{p}(G)$ which commute with translations. From Edwards' theorem and $\|\mu * g\|_{p} \leqq K\|g\|_{1}$ it follows that $\mu * g=f * g$ for all $g \in L_{1}(G)$ where $f \in L_{p}(G)$. It is a simple consequence that $\hat{\mu}=\hat{f}$.

\section{REFERENCES}

1. A. C. Berry, Necessary and sufficient conditions in the theory of Fourier transforms, Ann. of Math. 32 (1931), 830-838.

2. H. Cramér, On the represententation of a function by certain Fourier integrals, Trans. Amer. Math. Soc. 46 (1939), 191-201.

3.W. F. Eberlein, Characterization of Fourier-Stieltjes transforms, Duke Math. J. 22 (1955), 465-468.

4. R. E. Edwards, On factor functions, Pacific J. Math. 5 (1955), 376-378.

5. P. R. Halmos, Measure theory, Van Nostrand, NewYork, 1950.

6. H. Helson, Isomorphisms of Abelian group algebras, Ark. Mat. 2 (1953), 475-487.

7. E. Hewitt, A survey of abstract harmonic analysis, Surveys in Applied Mathematics Vol. IV, Wiley, New York, 1958.

8. L. H. Loomis, An introduction to abstract harmonic analysis, Van Nostrand, New York, 1953.

9. W. Rudin, Measure algebras on abelian groups, Bull. Amer. Math. Soc. 65 (1959), 227-247

10. R. Salem, Les coefficients de Fourier des functions sommables, C. R. Acad. Sci. Paris 192 (1931), 144-146.

11. —_ Essais sur les series trigonométriques, Hermann, Paris, 1940.

12. I. J. Schoenberg, A remark on the preceding note by Bochner, Bull. Amer. Math. Soc. 40 (1934), 277-278.

California Institute of Technology,

Pasadena, California 